\title{
BMJ Open Economic evaluation of intrahospital clinical practices in injury care: protocol for a 10-year systematic review
}

Blanchard Conombo (D) ,1,2 Jason Robert Guertin (D) , ${ }^{1}$ Pier-Alexandre Tardif, ${ }^{2}$ Imen Farhat, ${ }^{2}$ Thomas Moore, ${ }^{2}$ Samy Bouderba, ${ }^{2}$ Kahina Soltana, ${ }^{2}$ Patrick Archambault, ${ }^{3}$ Simon Berthelot, ${ }^{2,3}$ François Lauzier, ${ }^{2,4}$ Alexis F Turgeon (D) ,2,4 Henry Thomas Stelfox, ${ }^{5}$ Michaël Chasse, ${ }^{6}$ Jeffrey Hoch, ${ }^{7}$ Lynne Moore ${ }^{1,2}$

To cite: Conombo B, Guertin JR, Tardif P-A, et al. Economic evaluation of intrahospital clinical practices in injury care: protocol for a 10-year systematic review. BMJ Open 2020;10:e034472. doi:10.1136/ bmjopen-2019-034472

- Prepublication history and additional material for this paper are available online. To view these files, please visit the journal online (http://dx.doi. org/10.1136/bmjopen-2019034472).

Received 20 September 2019 Revised 14 April 2020 Accepted 28 May 2020
Check for updates

(C) Author(s) (or their employer(s)) 2020. Re-use permitted under CC BY-NC. No commercial re-use. See rights and permissions. Published by BMJ.

For numbered affiliations see end of article.

Correspondence to

Blanchard Conombo;

blanchard.conombo.1@ulaval.ca

\section{ABSTRACT}

Introduction Underuse of high-value clinical practices and overuse of low-value practices are major sources of inefficiencies in modern healthcare systems. Injuries are second only to cardiovascular disease in terms of acute care costs but data on the economic impact of clinical practices for injury admissions are lacking. This study aims to summarise evidence on the economic value of intrahospital clinical practices for injury care.

Methods and analysis We will perform a systematic review to identify research articles in economic evaluation of intrahospital clinical practices in acute injury care. We will search MEDLINE and databases such as Embase, Web of Science, NHS Economic Evaluation Database, Cochrane CENTRAL, BIOSIS and CINAHL for randomised or non-randomised controlled trials and observational studies using a combination of keywords and controlled vocabulary. We will consider the following outcomes relative to economic evaluations: incremental cost-effectiveness ratio, incremental cost-utility ratio, incremental net health benefit, incremental net monetary benefit (iNMB) and incremental cost-benefit ratio. Pairs of independent reviewers will evaluate studies that meet eligibility criteria and extract data from included articles using an electronic data extraction form. All outcomes will be converted into iNMB. We will report iNMB for practices classified by type of practice (hospitalisation, consultation, diagnostic, therapeuticsurgical, therapeutic-drugs, therapeutic-other). Results obtained with a ceiling ratio of $\$ 50000$ per qualityadjusted life year gained for identified clinical practices will be summarised by charting forest plots. In line with Cochrane recommendations for systematic reviews of economic evaluations, meta-analyses will not be conducted.

Ethics and dissemination Ethics approval is not required as original data will not be collected. This study will summarise existing evidence on the economic value of clinical practices in injury care. Results will be used to advance knowledge on value-based care for injury admissions and will be disseminated through a peerreviewed article, international scientific meetings and clinical and healthcare quality associations.
Strengths and limitations of this study

- Fill a major knowledge gap on the economic value of intrahospital clinical practices for acute injury care.

- Advance the agenda on value-based healthcare for injury admissions.

- Inform research priorities.

- Represents a crucial step towards the de-adoption of low-value clinical practices in acute injury care.

- For feasibility reasons, restricted to studies published in English since 2009.

\section{INTRODUCTION}

In Canada, injuries represent the leading cause of potential years of life lost and cost more than heart and stroke diseases combined. ${ }^{1}$ In 2035, the direct costs of injury are expected to reach $\$ \mathrm{C} 75$ billion while they were estimated at $\$ \mathrm{C} 27$ billion in 2007 , ${ }^{1}$ representing an increase of almost $200 \%$. $^{2}$ Injuries represent the third leading cause of potential years of life lost in the USA. ${ }^{3}$ The estimated total lifetime medical and work loss costs associated with fatal and non-fatal injuries in the USA were $\$ 671$ billion in 2013 . $^{4}$

Regional variations in injury outcomes between healthcare providers have been observed in Canada, the USA and the UK that are not explained by patient case mix..$^{5-7}$ This evidence of suboptimal injury outcomes suggests that efforts must be made to optimise clinical practices in injury care. ${ }^{8}$ Valuebased healthcare is defined as "care that is tailored for optimising health and wellbeing by delivering what is needed, wanted, clinically effective, affordable, equitable, and responsible in its use of resources". ${ }^{10}$ When patients do not receive tests and treatments that have been shown to be effective for their condition, we refer to underuse. ${ }^{11} \mathrm{Up}$ to $50 \%$ of patients admitted for injury do not receive 
recommended care. ${ }^{12}$ The economic impact of the underuse of recommended care implies missed opportunities of healthcare cost savings, averted productivity losses and the monetised value of potential reductions in morbidity and mortality. When patients undergo tests and treatments that are not supported by evidence and/or could expose them to unnecessary harm, they receive low-value care, widely referred to as overuse. ${ }^{13}$ Overuse is driven by low-value clinical practices, which consume up to $30 \%$ of healthcare resources and threaten the sustainability of affordable and accessible healthcare. ${ }^{14-17}$ From an economic evaluation standpoint, the overuse of low-value practices implies inefficiency in resources use that results in a waste of resources. More importantly, low-value practices expose patients to adverse events and delays to effective treatment. ${ }^{18}$ The estimated overuse of healthcare services in the USA amounts to $\$ 780$ billion annually. ${ }^{19}$

To achieve value-based care, guidelines and recommendations should target both underuse and overuse and be supported by data provided from economic evaluations. ${ }^{20}$ However, current guidelines on clinical practices in injury care focus almost exclusively on underuse and are rarely supported by evidence of cost-effectiveness. ${ }^{21-24}$ This systematic review aims to review evidence of the economic value of intrahospital clinical practices in acute injury care to advance knowledge on value-based care in this patient population.

\section{METHODS AND ANALYSIS}

The structure of the protocol follows the Preferred Reporting Items for Systematic Review and Meta-Analysis Protocols guidelines for systematic reviews. ${ }^{25}$ Any changes to the protocol will be documented in the final published report.

\section{Patient and public involvement}

No patient involved.

\section{Relevant studies}

Inclusion criteria

We will include research articles, systematic reviews, reports and guidelines on cost-effectiveness analyses (eg, cost per life year gained), cost-utility analyses (eg, cost per quality-adjusted life year (QALY) gained or cost per disability-adjusted life year averted), cost-benefit and costminimisation analyses of intrahospital clinical practices for patients treated in hospital for injury. Clinical practices could include admissions, transfers, consultations and diagnostic or therapeutic procedures. A 'do nothing' strategy, standard care or any other strategy will be considered as potential comparators.

The following outcomes of economic evaluation will be considered: incremental cost-effectiveness ratio ('ICER'), incremental cost-utility ratio ('ICUR'), incremental net monetary benefit ('iNMB'), incremental net health benefit ('iNHB') and the incremental cost-benefit ratio. Studies identifying the results of the economic evaluation as one clinical practice being dominant or dominated will be included. Such results would indicate that one comparator is less costly and more effective than the other. We will restrict the review to studies published in English in the last 10 years (from January 2009) to ensure feasibility of the review and results that are current.

\section{Exclusion criteria}

We will not include cost-consequences analyses, budget impact studies, narrative reviews, research protocols or conference abstracts. Studies providing incremental costs without incremental effectiveness or vice versa will not be included. Studies on experimental interventions, military injuries, cadavers or animals will be excluded. Studies in which there is no comparator group will be excluded. Our systematic review will be limited to evidence from high-income countries.

\section{Information sources}

We will search MEDLINE (via PubMed), EMBASE, Web of Science, NHS Economic Evaluation Database, Health Technology Assessment Database, EconLit, Tufts CEA Registry, Cochrane CENTRAL, BIOSIS and CINAHL to identify research articles on economic evaluation of clinical practices specific to intrahospital acute injury care. The grey literature will be searched through thesis repositories, injury association websites, healthcare quality websites and the Web of Knowledge. Thesis repositories include Thesis portal Canada, Electronic Thesis Online Service (EThOS), Digital Access to Research Theses (DART)-Europe E-Theses Portal and the National Library of Australia's Trove and ProQuest Dissertations \& Theses Global. Healthcare quality websites include the WHO, National Institute for Health and Care Excellence, National Association for Healthcare Quality, National Quality Forum, Lown Institute, Agency for Healthcare Research and Quality, Choosing Wisely, Canadian Institutes for Health Information, Australasian Association for Quality in Healthcare. Injury organisations include the American College of Surgeons, Trauma Association of Canada, International Association for Trauma Surgery and Intensive Care, Australasian Trauma Society, Trauma Audit Research Network, American Association for the Surgery of Trauma, Eastern Association for the Surgery of Trauma, American Trauma Society, British Trauma Society, Orthopaedic Trauma Association, Western Trauma Association, Trauma.org, The Society of Trauma Nurses, International Trauma Anaesthesia and Critical Care Society, the Brain Trauma Foundation and patient advocacy organisations including Safer Healthcare Now!

\section{Search strategy}

A rigorous strategy will be designed using a combination of Boolean terms with relevant keywords and subject headings covering 'injury', 'trauma' and 'economic evaluation' for EMBASE (EMBASE tree; EMTREE) and PubMed (Medical Subject Headings; MeSH), and then adapted to the remaining databases (see online 
supplementary appendix 1 for the preliminary search strategy of 28 December 2019). Clinicians in the project steering committee and information specialists will be consulted to refine the search strategy using the Peer Review of Electronic Search Strategies checklist. ${ }^{26}$ The sensitivity of our search strategy will be evaluated by identifying between 5 and 10 sentinel articles and checking whether they are detected.

\section{Select studies}

Data management

Citations will be managed using EndNote software (V.X7.0.1, New York City: Thomson Reuters, 2011). Duplicates will be identified and removed via electronic and manual screening. If multiple publications based on the same dataset are identified, we will select the most recent study or the one with the largest sample size.

\section{Selection process}

Pairs of independent reviewers (LM, BC, PAT, IF, TM, KS, SB) will screen all titles, abstracts and full texts to identify eligible studies. Prior to selection, we will evaluate interreviewer agreement on eligibility using the first 500 citations. Discrepancies between reviewers will be resolved by consensus. We will re-specify eligibility criteria if necessary and repeat the selection process until an acceptable interrater agreement is attained. A third reviewer will adjudicate if necessary (JRG). The level of agreement between reviewers will be assessed with Kappa coefficients ${ }^{27}$ and agreement will be considered acceptable if kappa $>0.9$. If information on eligibility is unavailable or unclear, study authors will be contacted.

\section{Chart material \\ Data collection}

An electronic data abstraction form will be developed with a detailed instruction manual and piloted on a representative sample of 10 publications. An example of the extraction grid is presented in online supplementary appendix 2. Pairs of reviewers with methodological and content expertise (BC, IF, PAT, MAG) will extract the following information from eligible articles: study design (systematic review, randomised controlled trial, observational study, simulation study), setting (country, year, hospital), type of economic evaluation (cost-effectiveness, cost-minimisation, cost-utility, costbenefit), perspective of economic evaluation (patient, hospital/clinic, healthcare system or societal), population (age, type of injury, injury severity, sample size), treatment and comparator, primary outcomes of the economic evaluation as stated above and authors' conclusions. Any discrepancies between reviewers will be resolved by consensus and a third reviewer will adjudicate if necessary (JRG). If important information is missing or requires clarification, we will contact study authors using up to three email attempts over 1 month to all listed authors.

\section{Collate, summarise and report on results}

Two reviewers (BC, MAG) will independently classify clinical practices according to the type of practice (hospitalisation, consultation, diagnostic, therapeutic-surgical, therapeutic-drugs, therapeutic-device, therapeuticother). Any disagreements will be adjudicated by a third reviewer (LM). Evidence of cost-effectiveness, cost-utility, cost-benefit or cost-minimisation (or lack of cost-effectiveness, cost-utility or cost-benefit) for clinical practices will be presented by the type and number of studies as well as measures of economic value. All measures will be converted into iNMB using a ceiling ratio (ie, the maximum acceptable willingness to pay per unit of health gain) of \$50 000 per QALY gained. We will use a conservative threshold of $\$ 50000$ per QALY gained because it is a widely used threshold in the literature for developed countries and using a single threshold will facilitate the comparison between studies. Measures of iNMB based on this threshold will represent a conservative estimate of incremental net monetary benefits. Results obtained with this ceiling ratio for identified clinical practices will be summarised by charting forest plots or league tables. We anticipate that meta-analyses will be inappropriate due to the heterogeneity of cost estimates both within and between settings. ${ }^{28}$

\section{Methodological quality of included studies}

Two content experts will independently assess methodological quality using the Consensus on Health Economic Criteria. $^{29}$

\section{ETHICS AND DISSEMINATION}

The results of this systematic review will fill a major knowledge gap on the economic value of clinical practices in acute injury care. They will be used to advance knowledge on value-based healthcare in this population. Results will be disseminated through a peer-reviewed article, international scientific meetings and clinical and healthcare quality associations. Ethics approval is not required as original data will not be collected.

\section{Author affiliations}

${ }^{1}$ Department of Social and Preventive Medicine, Laval University, Quebec City, Quebec, Canada

${ }^{2}$ Population Health and Optimal Health Practices Research Unit, Trauma Emergency - Critical Care Medicine, Centre de Recherche du CHU de Québec (Hôpital de l'Enfant-Jésus), Laval University, Quebec City, Quebec, Canada ${ }^{3}$ Department of Family Medicine and Emergency Medicine, Laval University, Quebec City, Quebec, Canada

${ }^{4}$ Department of Anesthesia and Critical Care Medicine, Laval University, Quebec City, Quebec, Canada

${ }^{5}$ Critical Care Medicine, University of Calgary, Calgary, Alberta, Canada

${ }^{6}$ Department of Medicine, University of Montreal, Montreal, Quebec, Canada ${ }^{7}$ Department of Public Health Sciences, University of California, Sacramento, California, USA

\section{Twitter Patrick Archambault @patarchambault}

Contributors BC led the development of the protocol and drafted the manuscript. JRG contributed to the development of research objectives and inclusion criteria, elaborated keywords, validated the data extraction form, critically revised the 
manuscript and approved the final version. P-AT contributed to the elaboration of keywords, developed and tested the search strategy, critically revised and approved the final version of the manuscript. IF contributed to the elaboration of keywords, developed and tested the search strategy, critically revised and approved the final version of the manuscript. TM contributed to the elaboration of keywords, developed and tested the search strategy, critically revised and approved the final version of the manuscript. SaB contributed to the elaboration of keywords, developed and tested the search strategy, critically revised and approved the final version of the manuscript. KS contributed to the elaboration of keywords, developed and tested the search strategy, critically revised and approved the final version of the manuscript. PA contributed to working definitions, developed keywords, revised the manuscript and approved the final version. SiB contributed to the development of research objectives and inclusion criteria, elaborated keywords, critically revised the manuscript and approved the final version. FL contributed to developing keywords, validated the search strategy and the data extraction form, revised the manuscript and approved the final version. AFT elaborated inclusion criteria and clinically significant outcomes, validated the search strategy, elaborated keywords, revised the manuscript and approved the final version. HTS contributed to the development of research objectives, inclusion criteria, the search strategy and the extraction form, developed keywords, revised the manuscript and approved the final version. MC validated the search strategy and the data extraction form, revised the manuscript and approved the final version. $\mathrm{JH}$ contributed to working definitions, developed keywords, revised the manuscript and approved the final version. LM led the development of the protocol and drafted the manuscript. She acts as guarantor for the review.

Funding This research was supported by the Canadian Institutes of Health Research (foundation grant number 353374 and Embedded Clinician Researcher $(\mathrm{PA}))$. LM, JRG, FL and MC are recipients of a research salary award from the Fonds de Recherche du Québec - Santé (FRQS). AFT is the Canada Research Chair in Critical Care Neurology and Trauma. The funders had no role in developing this protocol.

\section{Competing interests None declared.}

Patient and public involvement Patients and/or the public were not involved in the design, or conduct, or reporting or dissemination plans of this research.

Patient consent for publication Not required.

Provenance and peer review Not commissioned; externally peer reviewed.

Open access This is an open access article distributed in accordance with the Creative Commons Attribution Non Commercial (CC BY-NC 4.0) license, which permits others to distribute, remix, adapt, build upon this work non-commercially, and license their derivative works on different terms, provided the original work is properly cited, appropriate credit is given, any changes made indicated, and the use is non-commercial. See: http://creativecommons.org/licenses/by-nc/4.0/.

ORCID iDs

Blanchard Conombo http://orcid.org/0000-0001-6636-8164

Jason Robert Guertin http://orcid.org/0000-0003-1718-5307

Alexis F Turgeon http://orcid.org/0000-0001-5675-8791

\section{REFERENCES}

1 Annotated bibliography of trauma care systems categorization and regionalization. Ann Emerg Med 1986;15:133-5.

2 Parachute. The cost of injury in Canada; 2015.

3 What do we know about the burden of disease in the U.S.? 2017. Available: https://www.healthsystemtracker.org/chart-collection/ know-burden-disease-u-s/\#item-cancer-circulatory-diseasesleading-causes-years-life-lost-u-s

4 CDC. Cost of injury data, 2020. Available: https://www.cdc.gov/ injury/wisqars/cost/index.html
5 Birkmeyer JD, Reames BN, McCulloch P, et al. Understanding of regional variation in the use of surgery. Lancet 2013;382:1121-9.

6 Moore L, Evans D, Hameed SM, et al. Mortality in Canadian trauma systems: a multicenter cohort study. Ann Surg 2017;265:212-7.

7 Fisher ES, Wennberg DE, Stukel TA, et al. The implications of regional variations in Medicare spending. Part 1: the content, quality, and accessibility of care. Ann Intern Med 2003;138:273-87.

8 Moore L, Boukar KM, Tardif P-A, et al. Low-Value clinical practices in injury care: a scoping review protocol. BMJ Open 2017;7:e016024.

9 Elshaug AG, Rosenthal MB, Lavis JN, et al. Levers for addressing medical underuse and overuse: achieving high-value health care. Lancet 2017;390:191-202.

10 What is value-based healthcare? Available: https://catalyst.nejm.org/ what-is-value-based-healthcare/

11 Berwick DM. Avoiding overuse-the next quality frontier. Lancet 2017;390:102-4.

12 Bartlett G, Blais R, Tamblyn R, et al. Impact of patient communication problems on the risk of preventable adverse events in acute care settings. CMAJ 2008;178:1555-62.

13 Kleinert S, Horton R. From universal health coverage to right care for health. Lancet 2017;390:101-2.

14 Association médicale du Québec. Plus de cinq milliards MAL dépensés dans Le Système de santé: AMQ, 2013. Available: https://www.amq.ca/fr/publications/nos-communiques-de-presse/ communiques-2013/item/502-plus-de-cinq-milliards-mal-depensesdans-le-systeme-de-sante [Accessed 17 Sep 2018].

15 Rich P. Physicians taking lead on appropriateness of care: Canadian Medical association, 2013. Available: https://www.cma.ca/En/Pages/ Physicians-taking-lead-appropriateness-care.aspx [Accessed 17 Sep 2018].

16 Pasadena hospital to leave L.A. County trauma system. Mod Healthc 1990;20:2.

17 Reilly BM, Evans AT. Much ado about (doing) nothing. Ann Intern Med 2009;150:270-1.

18 Berwick DM, Hackbarth AD. Eliminating waste in US health care. JAMA 2012;307:1513-6.

19 Morlière $C$, Verpillot $E$, Donon L, et al. A cost-utility analysis of sacral anterior root stimulation (SARS) compared with medical treatment in patients with complete spinal cord injury with a neurogenic bladder. Spine J 2015;15:2472-83.

20 Gray M. Value based healthcare. BMJ 2017;356:j437.

21 American College of Surgeons. Resources for optimal care of the injured patient 2014/Resources Repository, 2014. Available: https:// www.facs.org/quality-programs/trauma/tqp/center-programs/vrc/ resources [Accessed 17 Sep 2018].

22 Carney N, Ghajar J, Jagoda A, et al. Concussion guidelines step 1: systematic review of prevalent indicators. Neurosurgery 2014;75 Suppl 1:S3-15.

23 Carney N, Totten AM, O'Reilly C, et al. Guidelines for the Management of Severe Traumatic Brain Injury, Fourth Edition. Neurosurgery 2017;80:6-15.

24 Eastern Association for the Surgery of Trauma. East practice management guidelines, 2018. Available: https://www.east.org/ education/practice-management-guidelines [Accessed 17 Sep 2018].

25 Preferred reporting items for systematic review and meta-analysis protocols (PRISMA-P) 2015 statement. Available: https://www. equator-network.org/reporting-guidelines/prisma-protocols/

26 McGowan J, Sampson M, Salzwedel DM, et al. PRESS Peer Review of Electronic Search Strategies: 2015 Guideline Statement. J Clin Epidemiol 2016;75:40-6.

27 Cohen J. A coefficient of agreement for nominal scales. Educ Psychol Meas 1960;20:37-46.

28 Higgins J, Green S. Cochrane Handbook for systematic reviews of interventions (15.6.3 meta-analysis of resource use and cost data), 2011. Available: https://handbook-5-1.cochrane.org/chapter_15/15 6_3_meta_analysis_of_resource_use_and_cost_data.htm [Accessed 16 Aug 2019].

29 Evers S, Goossens M, de Vet H, et al. Criteria list for assessment of methodological quality of economic evaluations: consensus on health economic criteria. Int J Technol Assess Health Care 2005;21:240-5. 